\title{
Hausdorff Discretization and Its Comparison to Other Discretization Schemes
}

\author{
Mohamed Tajine, Daniel Wagner and Christian Ronse \\ LSIIT UPRES-A ULP-CNRS 7005 \\ (Laboratoire des Sciences de l'Image, d'Informatique et de Télédétection), \\ Dépt. Informatique, 7, rue René Descartès, F67084 Strasbourg cedex \\ \{tajine, ronse\}@dpt-info.u-strasbg.fr
}

\begin{abstract}
We study the problem of discretization in a Hausdorff space followed in [WTR 98]. We recall the definitions and properties of the Hausdorff discretization of a compact set. We also study the relationship between the covering discretizations and the Hausdorff discretization. For a cellular metric every covering discretization minimizes the Hausdorff distance, and conversely, if the supercover discretization minimizes the Hausdorff distance then the metric is cellular. The supercover discretization is the Hausdorff discretization iff the metric is proportional to $d_{\infty}$. We compare also the Hausdorff discretization and the Bresenham discretization [Bres 65]. Actually, the Bresenham discretization of a segment of $\mathbb{R}^{2}$ is not always a good discretization relatively to a Hausdorff metric.
\end{abstract}

Keywords: Bresenham discretization, cellular metric, Hausdorff metric, lattice, covering discretization, Hausdorff discretization.

\section{Introduction}

Let $K$ be a compact subset of $\mathbb{R}^{n}$, we investigate the following problem : determine the set $\mathcal{M}_{H_{d}}(K)$ of subsets of $\mathbb{Z}^{n}$ minimizing a Hausdorff distance to $K$. We have shown [Wag 97] that $\mathcal{M}_{H_{d}}(K)$ is stable by union and that the union of all its elements is also a set minimizing the Hausdorff distance. This union is called the Hausdorff discretization of the compact $K$. We also proved that the "Digital geometry" converges in Hausdorff space to the "Euclidean geometry" as in [DT 95].

The supercover discretization operator $\Delta_{S C}$ is defined by $\forall K \subset \mathbb{R}^{n}, \Delta_{S C}(K)=$ $\left\{p \in \mathbb{Z}^{n} \mid K \cap \mathcal{C}(p) \neq \emptyset\right\}$ where for each $p, \mathcal{C}(p)$ is the cell corresponding to $p$. We also study the relationship between the covering discretizations and the Hausdorff discretization. We proved that, for a cellular metric every covering discretization minimizes the Hausdorff distance, and conversely, if the supercover discretization minimizes the Hausdorff distance then the metric is cellular. We proved also that the supercover discretization is the Hausdorff discretization iff the metric is proportional to $d_{\infty}$. We compare also the Hausdorff discretization 
and the Bresenham discretization [Bres 65]. Actually, the Bresenham discretization of a segment of $\mathbb{R}^{2}$ is not always a good discretization relatively to a Hausdorff metric.

This paper is divided in four sections. In the second section we present some classical notions of metric space and Hausdorff space, and we introduce some new metric notions. In the third section, we study the problem of discretization in a Hausdorff space and we introduce a new discretization : Hausdorff discretization. In the last section, we present some applications. Actually, we prove that the Bresenham discretization of a segment of $\mathbb{R}^{2}$ is not always a good discretization relatively to a Hausdorff metric.

The proofs are not given here, they will appear in future papers in preparation [RT 98]. Some of them can be found in [Wag 97].

\section{Metric space and Hausdorff space}

This section contains some classical notions of metric space and Hausdorff space, it also contains some new metric notions. The proofs of the classical notions used in this section can be found (for example) in [HY 88], [GK 90], [Bar 93].

Definition 1. Let $(\mathcal{E}, d)$ be a metric space and let $p \in \mathcal{E}$ and $r \in \mathbb{R}^{+}$,

$$
\mathcal{B}_{r}^{d}(p)=\{x \in \mathcal{E} \mid d(x, p) \leq r\} .
$$

Definition 2. Let $(\mathcal{E}, d)$ be a metric space and let $E \subseteq \mathcal{E}$, the interior of $E$ is the set $\operatorname{int}(E)=\left\{x \in E \mid \exists r>0, \mathcal{B}_{r}^{d}(p) \subset E\right\}$.

\subsection{Metric space and normed vector space}

Definition 3. Let $(\mathcal{E}, d)$ be a metric space and let $K \subseteq \mathcal{E} ; K$ is said to be compact if every infinite sequence in $K$ contains a subsequence having a limit in $K$.

Definition 4. Let $(\mathcal{E}, d)$ be a metric space and let $A \subset \mathcal{E}$ and $x_{0} \in \mathcal{E} ; d\left(x_{0}\right.$, $A)=i n f_{y \in A}\left(d\left(x_{0}, y\right)\right)$.

Definition 5. A metric $d$ on $\mathbb{R}^{n}$ is said to be invariant under translation if

$$
\forall(x, y, z) \in\left(\mathbb{R}^{n}\right)^{3}, d(x+z, y+z)=d(x, y) .
$$

Definition 6. A norm over a vector space $\mathcal{E}$ is an application $N: \mathcal{E} \longrightarrow \mathbb{R}^{+}$ such that:

$-\forall x \in \mathcal{E}, N(x)=0 \Leftrightarrow x=0$.

$-\forall x \in \mathcal{E}, \forall \lambda \in \mathbb{R}, N(\lambda x)=|\lambda| N(x)$.

$-\forall(x, y) \in \mathcal{E}^{2}, N(x+y) \leq N(x)+N(y)$.

$(\mathcal{E}, N)$ is called a normed vector space. 


\section{Remarks}

- If $N$ is a norm over $\mathcal{E}$, then the function $d_{N}$ such that : $\forall x, y \in \mathcal{E}, d_{N}(x$, $y)=N(x-y)$ is a metric over $\mathcal{E}$. $d_{N}$ is called the metric induced by the norm $N$.

- A metric induced by a norm is invariant under translation.

\section{Examples :}

$\mathcal{E}=\mathbb{R}^{n}$ and let $x=\left(x_{1}, x_{2}, \cdots, x_{n}\right) \in \mathbb{R}^{n}$.

$\forall p \geq 1, \quad\|x\|_{p}=\sqrt[p]{\left|x_{1}\right|^{p}+\cdots+\left|x_{n}\right|^{p}} \quad$ and $\quad\|x\|_{\infty}=\max _{1 \leq i \leq n}\left|x_{i}\right|=\lim _{p \rightarrow \infty}\|x\|_{p}$ are a norms over $\mathbb{R}^{n}$. The metrics induced by these norms are $d_{p}$ and $d_{\infty}$ respectively.

Property 1. [Sto 70]

- Let $N$ be a norm on $\mathbb{R}^{n}$, and let $B_{N}=\left\{x \in \mathbb{R}^{n} ; N(x) \leq 1\right\}$. Then $B_{N}$ is a compact convex set with a non-empty interior and symmetrical relatively to the origin.

- Conversely, for every compact convex set $K \subset \mathbb{R}^{n}$ of dimension $n$, which is symmetrical relatively to the origin, there is precisely one norm $N$ such that $B_{N}=K$. Moreover $\forall x \in \mathbb{R}^{n}, N(x)=\inf \left(\left\{r \in \mathbb{R}^{+} ; \frac{1}{r} x \in K\right\}\right)$

\subsection{Cellular metric}

In this subsection we introduce a new notion : a cellular metric. We present some properties of a cellular metric and we compare it with the metrics $d_{1}$ and $d_{\infty}$.

Definition 7. Let $p \in \mathbb{Z}^{n}$, we define the the cell of center $p$ as

$$
\mathcal{C}(p)=\left\{x \in \mathcal{E} \mid d_{\infty}(x, p) \leq \frac{1}{2}\right\}
$$

Definition 8. A metric $d$ over $\mathbb{R}^{n}$ is called cellular if $\forall x \in \mathbb{R}^{n}, \forall p, q \in \mathbb{Z}^{n}$, $x \in \mathcal{C}(p) \Longrightarrow d(p, x) \leq d(q, x)$. In particular, if $x \in \mathcal{C}(p) \cap \mathcal{C}(q)$, then $d(p$, $x)=d(q, x)$.

Definition 9. In $\mathbb{R}^{n}$, a symmetry relatively to the hyperplane $x_{i}=0$ is the function $\sigma_{i}: \mathbb{R}^{n} \rightarrow \mathbb{R}^{n}$ which associates to the vector $\left(x_{1}, \cdots, x_{n}\right)$ the vector $\left(x_{1}{ }^{\prime}, \cdots, x_{n}{ }^{\prime}\right)$ where $x_{i}{ }^{\prime}=-x_{i}$ and $x_{j}{ }^{\prime}=x_{j}$ for all $j \neq i$.

Proposition 1. Let $N$ be a norm invariant under $\sigma_{1}, \cdots, \sigma_{n}$, then the metric $d_{N}$ induced by $N$ is cellular.

Proposition 2. If $d$ is a cellular metric in $\mathbb{R}^{2}$ and $d$ is induced by a norm $N$, then $N$ is invariant under $\sigma_{1}$ and $\sigma_{2}$.

Definition 10. Let $d$ be a metric on $\mathbb{R}^{n}$ invariant by translation.

- The cellular covering radius of the metric $d$ is $r_{c c}(d)=\inf (\{r>0, \mathcal{C}(O) \subseteq$ $\left.\mathcal{B}_{r}^{d}(O)\right\}$ ) where $O$ is the origin.

- The covering radius of the metric d is $r_{c}(d)=\inf \left(\left\{r>0 \mid \mathbb{R}^{n}=\bigcup_{p \in \mathbb{Z}^{n}} \mathcal{B}_{r}^{d}(p)\right\}\right)$ 


\section{Remarks}

- $\left.\mathbb{R}^{n}=\bigcup_{p \in \mathbb{Z}^{n}} \mathcal{B}_{r_{c}(d)}^{d}(p)\right)$.

- $r_{c}(d) \leq r_{c c}(d)$.

Proposition 3. Let $d$ be a metric invariant by translation. If $d$ is cellular then $r_{c}(d)=r_{c c}(d)$.

Proposition 4. If $d$ is a metric induced by a norm $N$ invariant under $\sigma_{1}, \cdots$, $\sigma_{n}$, then $r_{c}(d)=N\left(\frac{1}{2}, \frac{1}{2}, \cdots, \frac{1}{2}\right)=\frac{1}{2} N(1,1, \cdots, 1)$.

Examples : In $\mathbb{R}^{n}, \forall p \geq 1, r_{c}\left(d_{p}\right)=\left(\frac{n^{\frac{1}{p}}}{2}\right)$, and $r_{c}\left(d_{\infty}\right)=\frac{1}{2}$.

Corollary 1. If $d$ is a metric induced by a norm $N$ and $d$ is invariant under $\sigma_{1}, \cdots, \sigma_{n}$, then $\mathcal{B}_{\frac{1}{2}}^{d_{\infty}}(O) \subseteq \mathcal{B}_{r_{c}(d)}^{d}(O)$

We introduce now some restrictions in order to compare the metric $d$ to the metric $d_{1}$.

Let $\mathcal{J}$ be a group of isometries of $\mathbb{R}^{n}$ (for the euclidean norm) preserving the canonical basis $\left\{e_{1}, \cdots, e_{n}\right\}$ : the elements of $\mathcal{J}$ are the linear applications whose the matrix is a permutation matrix (with coefficients in $\{0,1\}$, and exactly one "1" by line and exactly one "1" by column).

Let $\mathcal{G}(N)$ be the group of isometries in $\mathcal{J}$ for which $N$ is invariant :

$\mathcal{G}(N)=\left\{\psi \in \mathcal{J} \mid \forall x \in \mathbb{R}^{n}, \quad N(\psi(x))=N(x)\right\}$

Proposition 5. Let $N$ be a norm on $\mathbb{R}^{n}$ invariant under $\sigma_{1}, \cdots, \sigma_{n}$. If $\mathcal{G}(N)$ is transitive on the basis $\left\{e_{1}, \cdots, e_{n}\right\}$, (i.e. $\forall i, j \in\{1, \cdots, n\}, \exists \psi \in \mathcal{G}(N)$, $\left.\psi\left(e_{i}\right)=e_{j}\right)$, then

$$
\mathcal{B}_{\frac{1}{2}}^{d_{\infty}}(O) \subseteq \mathcal{B}_{r_{c}(d)}^{d}(O) \subseteq \mathcal{B}_{\frac{n}{2}}^{d_{1}}(O)
$$

Remarks : With the same hypothesis as in Proposition 5, we have :

Let $p, q \in \mathbb{Z}^{n}$.

- If $d_{1}(p, q)>n$, then $\mathcal{B}_{r_{c}(d)}^{d}(p) \cap \mathcal{B}_{r_{c}(d)}^{d}(q)=\emptyset$.

- If $d_{1}(p, q)=n$, then $\mathcal{B}_{r_{c}(d)}^{d}(p) \cap \mathcal{B}_{r_{c}(d)}^{d}(q) \cap \mathcal{C}(p)=\mathcal{C}(p) \cap \mathcal{C}(q)$, which is nonempty iff $p$ and $q$ are diagonally adjacents except for $d=\alpha d_{1}$.

\subsection{Hausdorff metric}

The definitions and results presenting in this subsection can be found in [GK 90], [Bar 93].

Definition 11. Let $(\mathcal{E}, d)$ be a metric space, $\mathcal{H}(\mathcal{E})$ is the set of the nonempty compact subsets of $\mathcal{E}$.

On $\mathcal{H}(\mathcal{E})$, we will define a metric $H_{d}$, such that if $(\mathcal{E}, d)$ is a complete metric space then $\left(\mathcal{H}(\mathcal{E}), H_{d}\right)$ is a complete metric space. 
Definition 12. Let $(\mathcal{E}, d)$ be a metric space. Let $A, B \in \mathcal{H}(\mathcal{E})$. We define the oriented Hausdorff metric from a set $A \in \mathcal{H}(\mathcal{E})$ to a set $B \in \mathcal{H}(\mathcal{E})$ by :

$h_{d}(A, B)=\max _{a \in A}\left(\min _{b \in B}(d(a, b))\right)=\max _{a \in A}(d(a, B))$

Definition 13. Let $(\mathcal{E}, d)$ be a metric space. The Hausdorff distance between two compact sets $A, B \in \mathcal{H}(\mathcal{E})$ is defined by $H_{d}(A, B)=\max \left(h_{d}(A, B), h_{d}(B, A)\right)$

Definition 14. Let $A, B$ be subsets of $\mathbb{R}^{n}$; the Minkowski addition of $A$ and $B$ is $A \oplus B=\{a+b \mid a \in A, b \in B\}=\bigcup_{a \in A} B(a)=\bigcup_{b \in B} A(b)$ where $\forall t \in \mathbb{R}^{n}$, $A(t)=A \oplus\{t\}$ is the translation of $A$ by $t$.

Property 2. Let $d$ be a metric on $\mathbb{R}^{n}$ and let $A, B \in \mathcal{H}\left(\mathbb{R}^{n}\right)$,

$H_{d}(A, B)=\min \left(\left\{r \geq 0 \mid A \subseteq \bigcup_{b \in B} \mathcal{B}_{r}^{d}(b)\right.\right.$ and $\left.\left.B \subseteq \bigcup_{a \in A} \mathcal{B}_{r}^{d}(a)\right\}\right)$. So, if the metric $d$ is invariant under translation, then

$\forall A, B \in \mathcal{H}\left(\mathbb{R}^{n}\right), \quad H_{d}(A, B)=\min \left(\left\{r \geq 0 \mid A \subseteq B \oplus \mathcal{B}_{r}^{d}(O)\right.\right.$ and $B \subseteq$ $\left.\left.A \oplus \mathcal{B}_{r}^{d}(O)\right\}\right)$.

\section{Property 3. [GK 90], [Bar 93]}

Let $(\mathcal{E}, d)$ be a metric space such that $d$ is invariant by translation :

- $\forall(A, B) \in \mathcal{H}(\mathcal{E})^{2}, \forall r \geq 0, h_{d}(A, B) \leq r \Longleftrightarrow A \subseteq B \oplus \mathcal{B}_{r}^{d}(0)$.

- $\forall(A, B, C, D) \in \mathcal{H}(\mathcal{E})^{4}, H_{d}(A \cup B, C \cup D) \leq \max \left(H_{d}(A, C), H_{d}(B, D)\right)$.

- $(\mathcal{E}, d)$ is a complete metric space $\Longleftrightarrow\left(\mathcal{H}(\mathcal{E}), H_{d}\right)$ is a complete metric space.

\section{Hausdorff Discretization}

We recall here the results presented in [WTR 97].

In all the following we assume that we have as metric space $\left(\mathbb{R}^{n}, d\right)$, where $d$ is a metric induced by a norm on $\mathbb{R}^{n}$, and as a discrete space $\mathbb{Z}^{n}$.

\subsection{Morphological discretization}

In this subsection we introduce the morphological discretization studied in [Hei 91a], [Hei 91b] and [Hei 92].

Definition 15. Let $K \subseteq \mathbb{R}^{n}$ and $\mathcal{S} \subseteq \mathbb{R}^{n}$, the discretization by dilation of $K$ by $\mathcal{S}$ is the set $\Delta_{\oplus}^{\mathcal{S}}(K)=(K \oplus \overline{\mathcal{S}}) \cap \mathbb{Z}^{n}$ where $\overline{\mathcal{S}}=\{-s \mid s \in \mathcal{S}\}$. $\mathcal{S}$ is called the structuring element.

Property 4. Let $K \subseteq \mathbb{R}^{n}$ and let $\mathcal{S} \subseteq \mathbb{R}^{n}$, then $\Delta_{\oplus}^{\mathcal{S}}(K)=\left\{p \in \mathbb{Z}^{n} \mid K \cap \mathcal{S}(p) \neq\right.$ $\emptyset\}$.

\section{Remarks, definitions and notations:}

Let $K \subseteq \mathbb{R}^{n}, d$ be a metric induced by a norm, and let $S \subseteq \mathbb{R}^{n}$ :

- Let $r \in \mathbb{R}^{+}$. For $S=\mathcal{B}_{r}^{d}(O), \Delta_{\oplus}^{\mathcal{S}}(K)$ is called the discretization of $K$ of radius

$r$ for the metric $d$ and it is denoted in the following by $\Delta_{r}^{d}(K)$. 
- For $S=\{0\}, \Delta_{\oplus}^{\mathcal{S}}(K)$ is the discretization, by sampling, of $K$.

- For $r=r_{c c}(d), \Delta_{r}^{d}(K)$ is called the discretization, by overlapping, of $K$.

- For $d=d_{\infty}, \Delta_{r_{c c}(d)}^{d}(K)=\left\{p \in \mathbb{Z}^{n} \mid \mathcal{C}(p) \cap K \neq \emptyset\right\}$ is the supercover discretization of $K$ [CK 95] which is denoted in the following by $\Delta_{S C}(K)$.

- Let $S \subset \mathbb{Z}^{n}$, if $\forall p \in S, K \cap \mathcal{C}(p) \neq \emptyset$ and $K \subseteq \bigcup_{p \in S} \mathcal{C}(p)$ then $S$ is called a covering discretization of $K$ [And 98]. So, the popular supercover discretization is the maximal covering discretization.

\subsection{Characterization of Hausdorff Discretization}

In this subsection we characterize the set $\mathcal{M}_{H_{d}}(K)$ of subset of $\mathbb{Z}^{n}$ minimizing the Hausdorff distance to a given compact $K$, we have proved [Wag 97] that $\mathcal{M}_{H_{d}}(K)$ is stable by union and that the union of all its elements is also a set minimizing the Hausdorff distance.

Definition 16. Let $K \in \mathcal{H}\left(\mathbb{R}^{n}\right)$. A set $M \subseteq \mathbb{Z}^{n}$ is a Hausdorff discretizing set of $K$ if $H_{d}(K, M)=\inf \left(\left\{H_{d}(K, S) \mid S \subseteq \mathbb{Z}^{n}\right\}\right)$.

- The value $r_{H}(K, d)=\inf \left(\left\{H_{d}(K, S) \mid S \subseteq \mathbb{Z}^{n}\right\}\right)$ is called the Hausdorff radius of the compact set $K$ for the metric $d$,

- $\mathcal{M}_{H_{d}}(K)=\left\{M \subseteq \mathbb{Z}^{n} \mid H_{d}(K, M)=\inf \left(\left\{H_{d}(K, S) \mid S \subseteq \mathbb{Z}^{n}\right\}\right)\right\}$ is the set of subsets of $\mathbb{Z}^{n}$ minimizing the Hausdorff distance to $K$.

- $\Delta_{H_{d}}(K)=\left(\bigcup_{M \in \mathcal{M}_{H_{d}}(K)} M\right)$ is called the Hausdorff discretization of $K$.

Property 5. Let $K \in \mathcal{H}\left(\mathbb{R}^{n}\right)$; then

- $\mathcal{M}_{H_{d}}(K)$ is non void and finite,

- $\forall M_{1}, M_{2} \in \mathcal{M}_{H_{d}}(K), M_{1} \cup M_{2} \in \mathcal{M}_{H_{d}}(K)$ and

- $\Delta_{H_{d}}(K) \in \mathcal{M}_{H_{d}}(K)$.

We will now characterize the Hausdorff discretization.

Theorem 1. Let $K \in \mathcal{H}\left(\mathbb{R}^{n}\right)$, then

- $r_{H}(K, d)=\inf \left(\left\{r \geq 0 \mid K \subseteq \bigcup_{p \in \Delta_{r}^{d}(K)} \mathcal{B}_{r}^{d}(p)\right\}\right)$,

- $\Delta_{H_{d}}(K)=\Delta_{r_{H}(K, d)}^{d}(K)=\left\{p \in D \mid K \cap \mathcal{B}_{r_{H}(K, d)}^{d}(p) \neq \emptyset\right\}$ and

- $r_{H}(K, d) \leq r_{c}(d)$.

Property 6. Let $K \in \mathcal{H}\left(\mathbb{R}^{n}\right), r \in \mathbb{R}^{+}$and let $S \subseteq \mathbb{Z}^{n}$ such that $K \subseteq \bigcup_{p \in S} \mathcal{B}_{r}^{d}(p)$ and $\forall p \in S, \quad \mathcal{B}_{r}^{d}(p) \cap K \neq \emptyset$, then $H_{d}(K, S) \leq r$. So, if $r=r_{H}(K, d)$ then $S \in \mathcal{M}_{H_{d}}(K)$.

Corollary 2. Let $d$ be a cellular metric. Then for every compact set $K$, if $S$ is a covering discretization of $K$ then $S \in \mathcal{M}_{H_{d}}(K)$.

The results obtained for the Hausdorff discretization in the lattice $\mathbb{Z}^{n}$ can be extended, in a natural way, to any lattice. In the following theorem, we prove that, the "Digital geometry" converges in Hausdorff space to the "Euclidean geometry" as in [DT 95] by using lattices with increasing resolution. 
Definition 17. Let $m \in \mathbb{N}$, the square lattice with step $\frac{1}{2^{m}}$ is the set $\mathbf{R}_{m}=$ $\frac{1}{2^{m}} \mathbb{Z}^{n}$.

Proposition 6. Let $K \in \mathcal{H}\left(\mathbb{R}^{n}\right)$. If $\forall m \in \mathbb{N}, \Delta_{H_{d}}\left(K, \mathbf{R}_{m}\right)$ is the Hausdorff discretization of $K$ in the lattice $\mathbf{R}_{m}$, then $\lim _{n \rightarrow \infty} H_{d}\left(K, \Delta_{H_{d}}\left(K, \mathbf{R}_{m}\right)\right)=0$.

\subsection{Hausdorff discretization and supercover discretization}

The supercover discretization operator $\Delta_{S C}$ is defined by

$$
\forall K \in \mathcal{H}\left(\mathbb{R}^{n}\right), \quad \Delta_{S C}(K)=\left\{p \in \mathbb{Z}^{n} \mid K \cap \mathcal{C}(p) \neq \emptyset\right\} .
$$

In this subsection, we study the relationship between the supercover discretization and the Hausdorff discretization. We present a localization of the Hausdorff discretization. We have shown that the supercover discretization minimizes the Hausdoff distance iff the metric is cellular, and we prove also that the supercover discretization is the Hausdorff discretization iff the metric is proportional to $d_{\infty}$.

Proposition 7. Let $d$ be a metric induced by a norm, then $\forall K \in \mathcal{H}\left(\mathbb{R}^{n}\right)$, $\Delta_{H_{d}}(K) \subseteq \Delta_{S C}(K) \oplus \Delta_{S C}\left(\mathcal{B}_{r_{c c}(d)}^{d}(O)\right)$.

Theorem 2. Let $d$ be a metric induced by a norm on $\mathbb{R}^{n}$.

- $d$ is a cellular $\Longleftrightarrow \forall K \in \mathcal{H}\left(\mathbb{R}^{n}\right), \quad \Delta_{S C}(K) \in \mathcal{M}_{H_{d}}(K)$.

- $\forall K \in \mathcal{H}\left(\mathbb{R}^{n}\right), \Delta_{H_{d}}(K)=\Delta_{S C}(K) \Longleftrightarrow \exists \alpha \in \mathbb{R}^{+}$such that $d=\alpha d_{\infty}$.

The topological proporties of the supercover discretization are studed by several people. [Sch 98] shows that, given a Euclidean set $K$ of $\mathbb{R}^{2}$, under some conditions on $k$, points can be removed from its supercover discretization $\Delta_{S C}(K)$, in such a way that, for the remaining subset $S$ of points, $\bigcup_{p \in S} \mathcal{C}(p)$ is homotopic to $K$. [LCG 98] gives a sufficient conditions on $K$ under wich $\Delta_{S C}(K)$ is homeomorphic to $K$.

\section{Applications}

In all the following, we assume that all the metrics considered are induced by a homogeneous norm.

Definition 18. A norm $N$ on $\mathbb{R}^{n}$ is homogeneous if $\forall\left(x_{1}, \cdots, x_{n}\right) \in \mathbb{R}^{n}$, $\forall\left(\epsilon_{1}, \cdots, \epsilon_{n}\right) \in\{-1,1\}^{n}$, for all permutations $\sigma, N\left(x_{1}, \cdots, x_{n}\right)=N\left(\epsilon_{1} x_{1}, \cdots\right.$, $\left.\epsilon_{n} x_{n}\right)=N\left(x_{\sigma(1)}, \cdots, x_{\sigma(n)}\right)$. So, if $n=2$, then $N$ is homogeneous iff $\forall\left(x_{1}\right.$, $\left.x_{2}\right) \in \mathbb{R}^{2}, N\left(x_{1}, x_{2}\right)=N\left(-x_{1}, x_{2}\right)=N\left(x_{2}, x_{1}\right)$.

Definition 19. Let $d$ be a metric on $\mathbb{R}^{n}$ and let $p \in \mathbb{R}^{n}$ and $r \in \mathbb{R}^{+}$,

$$
C_{r}^{d}(p)=\left\{x \in \mathbb{R}^{n} \mid d(x, p)=r\right\} .
$$


Property 7. Let $d$ be a metric on $\mathbb{R}^{n}$ induced by a homogeneous norm, then

- $\frac{1}{2}\{-1,1\}^{n} \subseteq\left(C_{r_{c}(d)}^{d}(p) \cap C_{1}^{d_{\infty}}(p)\right)$ and

- $\frac{1}{2}\{-1,1\}^{n} \subset\left(C_{r_{c}(d)}^{d}(p) \cap C_{1}^{d_{\infty}}(p)\right) \Longleftrightarrow \exists \alpha \in \mathbb{R}^{+}$such that $d=\alpha d_{\infty}$.

- If $K \in \mathcal{H}\left(\mathbb{R}^{n}\right)$, then $r_{H}(K, d)=r_{c}(d) \Longleftrightarrow K \cap\left(\left\{\left(\frac{1}{2}, \cdots, \frac{1}{2}\right)\right\} \oplus \mathbb{Z}^{n}\right) \neq \emptyset$

\section{Notations :}

Let $d$ be a metric on $\mathbb{R}^{2}$, and let $p \in \mathbb{Z}^{2}$.

- $\mathcal{N}(p)=\mathbb{R}^{2} \backslash\left(\bigcup_{q \in\left(\mathbb{Z}^{2} \backslash\{p\}\right)} \mathcal{B}_{r_{c}(d)}^{d}(q)\right)$,

- $\mathcal{V}_{8}(p)=\left\{q \in \mathbb{Z}^{2} \mid d_{\infty}(p, q)=1\right\}$ and

- $\mathcal{V}_{4}(p)=\left\{q \in \mathbb{Z}^{2} \mid d_{1}(p, q)=1\right\}$.

$\mathcal{N}(p), \mathcal{V}_{4}(p)$ and $\mathcal{V}_{8}(p)$ are illustrates in the Figures $1,2$.

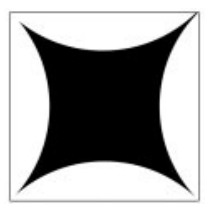

Fig. 1. $\mathcal{N}(O)$ for the metric $d_{2}$.

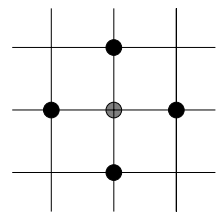

( a )

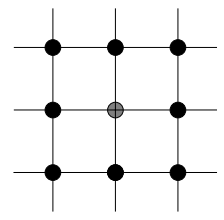

( b )

Fig. 2. (a) correspond to $\mathcal{V}_{4}(O)$, (b) correspond to $\mathcal{V}_{8}(O)$

Property 8 . Let $d$ be a metric on $\mathbb{R}^{2}$ induced by a homogeneous norm, and let $p \neq q \in \mathbb{Z}^{2}$, and $r \in \mathbb{R}^{+}$.

- If $r<r_{c}(d)$, then $\left(\mathcal{B}_{r}^{d}(p) \cap \mathcal{B}_{r}^{d}(p) \neq \emptyset \Longrightarrow q \in \mathcal{V}_{4}(p)\right)$,

- If $r=r_{c}(d)$, then $\left(\mathcal{B}_{r}^{d}(p) \cap \mathcal{B}_{r}^{d}(q) \neq \emptyset \Longrightarrow q \in \mathcal{V}_{8}(p)\right)$.

Property 9. Let $d$ be a metric on $\mathbb{R}^{2}$ induced by a homogeneous norm, and let $p \in \mathbb{Z}^{n}$, then $\mathcal{N}(O)$ is an open subset of $\mathbb{R}^{n}$ and $(\mathcal{N}(O)=\emptyset \Longleftrightarrow \exists$ $\alpha \in \mathbb{R}^{+}$such that $\left.d=\alpha d_{1}\right)$.

Definition 20. Let $d$ be a metric on $\mathbb{R}^{n}$ and $K \in \mathcal{H}\left(\mathbb{R}^{n}\right)$, the skeleton of $\Delta_{H_{d}}(K)$ is the set

$$
\mathcal{S} k(K, d)=\left\{q \in \mathbb{Z}^{n} \mid \forall S \subset \mathbb{Z}^{n}, H_{d}(K, S)=r_{H}(K, d) \Longrightarrow q \in S\right\}
$$


Definition 21. Let $K$ be a connected subset of $\mathbb{R}^{2}$ and $\mathcal{S}$ a square of $\mathbb{R}^{2}$, we say that $K$ goes throught $\mathcal{S}$ if $\exists p, q \in \mathbb{R}^{2}$ such that $p \neq q, p, q \in(K \cap(\mathcal{S} \backslash$ int $(\mathcal{S}))$ and $[p, q] \cap \operatorname{int}(\mathcal{S}) \neq \emptyset$, where $[p, q]=\{t p+(1-t) q \mid 0 \leq t \leq 1\}$ is the segment of $\mathbb{R}^{2}$ of end points $p$, .

Figure 3 present a curve goes throught a square and a curve not goes throught a square.

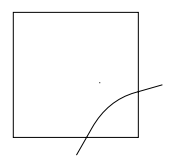

(a)

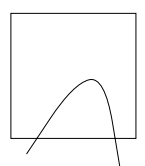

(b)

Fig. 3. in (a) the curve goes throught the square,

in (b) the curve not goes throught the square

Property 10. Let $d$ be a metric on $\mathbb{R}^{2}$ induced by a homogeneous norm, and let $K \in \mathcal{H}\left(\mathbb{R}^{2}\right)$. If $K$ is connected and $r_{H}(K, d)<r_{c}(d)$, then

$$
K \text { goes through } \mathcal{C}(p) \Longrightarrow p \in \mathcal{S} k(K, d)
$$

\subsection{Hausdorff discretization and Bresenham discretization}

In this subsection, we compare the Hausdorff and Bresenham discretizations [Bres 65]. Actually, we prove that the Bresenham discretization of a segment of $\mathbb{R}^{2}$ is not always a good discretization relatively to a Hausdorff metric.

In all the following, we consider only the segments of $\mathbb{R}^{2}$ of the form $\{(x, a x+$ b) $\mid x \in[\alpha, \beta]\}$ where $|a| \leq 1, \alpha, \beta \in \mathbb{R}$ and $\beta-\alpha \geq 3$.

Definition 22. [Bre 65]

Let $\mathcal{L}$ be a segment of $\mathbb{R}^{2}$

$\Delta_{\text {Bres }}(\mathcal{L})=\left\{\left(i,\left\lfloor a i+b+\frac{1}{2}\right\rfloor\right) \quad \mid\lfloor\alpha\rfloor \leq i \leq\lceil\beta\rceil\right\}$ where $(\forall x \in \mathbb{R},\lfloor x\rfloor$, $\lceil x\rceil \in \mathbb{Z}, \quad\lfloor x\rfloor \leq x<x+1$ and $\lceil x\rceil-1<x \leq\lceil x\rceil) . \Delta_{\text {Bres }}(\mathcal{L})$ is called $a$ Bresenham discretization of the segment $\mathcal{L}$.

Definition 23. Let $d$ be a metric on $\mathbb{R}^{2}$ induced by a homogeneous norm, and let $E=\left\{(x, y) \in \mathcal{B}_{r_{c}(d)}^{d}(O) \mid y=-x+1\right\}$.

- $\mathcal{A}(d)$ is the point of $E$ of minimal $x$-coordinate (i.e. if $\mathcal{A}(d)=\left(x_{0}, y_{0}\right)$, then $\left.x_{0}=\min (\{x \mid(x,-x+1) \in E\})\right)$

- $\mathcal{R}(d)$ is the square with the set of vertex $V(\mathcal{R})=\left\{\left(x_{0}, y_{0}-1\right),\left(-x_{0}, y_{0}-1\right)\right.$, $\left.\left(x_{0}, 1-y_{0}\right),\left(-x_{0}, 1-y_{0}\right)\right\}$ (i.e. $\mathcal{R}(d)$ is the convex hull of $\left.V(\mathcal{R})\right)$ 
$\mathcal{A}(d)$ and $\mathcal{R}(d)$ are illustrates in Figure 4.

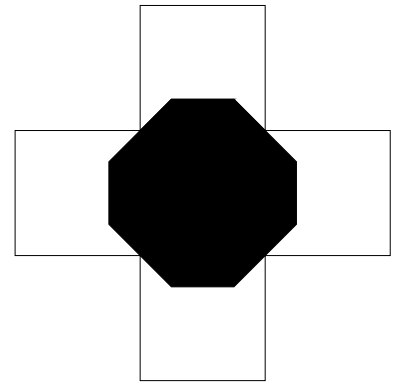

( a )

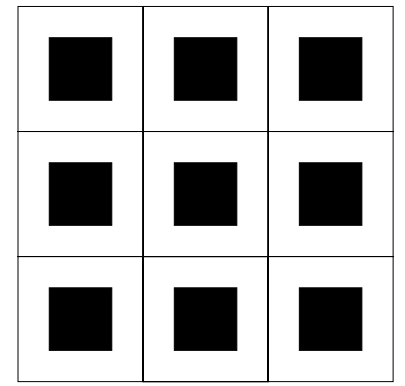

(b)

Fig. 4. (a) The black area represents $\mathcal{A}(d)$, (b) The black areas represent $\mathcal{R}(d) s$.

Property 11. Let $d$ be a metric on $\mathbb{R}^{2}$ induced by a homogeneous norm, and let $\mathcal{L}$ be a segment in $\mathbb{R}^{2}$. If $r_{H}(\mathcal{L}, d)=r_{c}(d)$, and $\mathcal{L}$ goes through $\mathcal{C}(p)$, then

$$
p \in \mathcal{S} k(\mathcal{L}, d) \Longleftrightarrow \mathcal{L} \cap(\{p\} \oplus \operatorname{int}(\mathcal{R}(d))) \neq \emptyset
$$

where $\operatorname{int}(\mathcal{R}(d))$ is the interior of the set $\mathcal{R}(d)$.

Theorem 3. Let $d$ be a metric on $\mathbb{R}^{2}$ induced by a homogeneous norm, and let $\mathcal{L}=\{(x, a x+b) \mid x \in[\alpha, \beta]\}$ be a segment in $\mathbb{R}^{2}$.

- If $r_{H}(\mathcal{L}, d)<r_{c}(d)$, then

$H_{d}\left(\Delta_{B r e s}(\mathcal{L}), \mathcal{L}\right)>r_{H}(\mathcal{L}, d) \Longleftrightarrow \exists p, q, r \in \mathbb{R}^{2} q, r \in \mathcal{V}_{4}(p), \quad r \in\left(\mathcal{V}_{8}(q) \backslash\right.$ $\left.\mathcal{V}_{4}(q)\right)$ and $\mathcal{L}$ goes throught $\mathcal{C}(p), \mathcal{C}(q), \mathcal{C}(r)$.

- If $r_{H}(\mathcal{L}, d)=r_{c}(d)$, then

$H_{d}\left(\Delta_{\text {Bres }}(\mathcal{L}), \mathcal{L}\right)>r_{H}(\mathcal{L}, d) \Longleftrightarrow \exists p, q, r \in \mathbb{R}^{2} q, r \in \mathcal{V}_{4}(p), \quad r \in\left(\mathcal{V}_{8}(q) \backslash\right.$ $\left.\mathcal{V}_{4}(q)\right)$ and $\mathcal{L}$ goes throught $\{p\} \oplus \mathcal{R}(d),\{q\} \oplus \mathcal{R}(d),\{r\} \oplus \mathcal{R}(d)$.

A different cases of Theorem 12 are illustrated in the Figures 5, 6 .

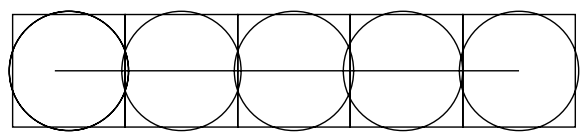

( a )

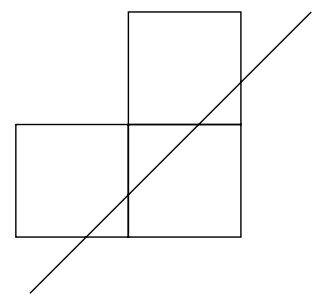

( b )

Fig. 5. (a) and (b) illustrates a different cases of theorem 12 where $r_{H}(\mathcal{L}, d)<r_{c}(d)$. 


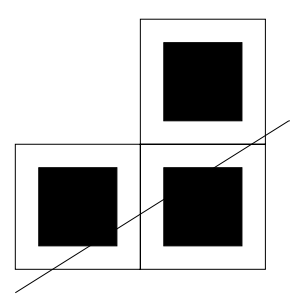

(a)

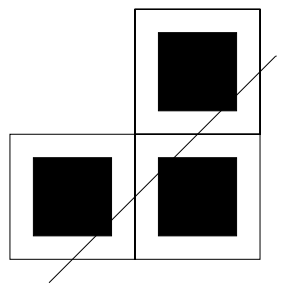

(b)

Fig. 6. (a) and (b) illustrates a different cases of theorem 12 where $r_{H}(\mathcal{L}, d)=r_{c}(d)$.

\section{Conclusion}

We have introduced a new theory of discretization, based on the Hausdorff distance. We have proved the convergence (in Hausdorff metric sens) of the discretization to the original object when resolution tends to zero. We have compared the Hausdorff discretization to other discretization schemes such as the covering, the supercover and the Bresenham discretization. We have proved that the supercover discretization is Hausdorff discretization for all compact set $k$ (i.e. $\left.\Delta_{S C}(K) \in \mathcal{M}_{H_{d}}(K)\right)$ iff the distance is cellular; and that for a distance $d$ induced by a norm, $\Delta_{H_{d}}(K)=\Delta_{S C}(K)$ for any compact set $K$ iff the distance $d$ is proportional to $d_{\infty}$. We have olso proved that Bresenham discretization of a segment of $\mathbb{R}^{2}$ is not always a good discretization relatively to a Hausdorff metric.

Further investigations will be needed:

- on the topological properties of Hausdorff discretization (cfr. [LCG 98], [Sch 98] );

- on its extension to grey-level images;

- on the discretization of geometrical and morphological operations.

\section{References}

[And 98] E. Andrès, Covering discretizations, Personal communication, 1998.

[Bar 93] M. F. Barnsley, Fractals Everywhere, Second Edition, Academic Press Professional, 1993.

[Bre 65] J. E. Bresenham, Algorithms for Computer Control of Digital Plotter, IBM Systems Journal, 4(1), pp25-30, 1965.

[CK 95] D. Cohen-or, A. Kaufman, Fundamentals of surface voxelization. Graphical Models and Image Processing, 57(6), pp 453-461, 1995.

[DT 95] S. Duval, M. Tajine, Digital geometry and Fractal Geometry, 5th Coll. DGCI'95, pp. 93-106, 1995.

[GK 90] K. Goebel, W.A. Kirk, Topics in metric fixed point theory., Cambridge Univ. Press, 1990.

[Hei 91a] H. J. A. M. Heijmans, Morphological Discretization, Geometrical Problem in Image Processing, U. Eckhardt and al. Eds, Akademie Verlag, Berlin, pp. 99-106, 1991. 
[Hei 91b] H. J. A. M. Heijmans, A. Toet, Morphological Sampling, Computer Vision, Graphics and Image Processing: Image Understanding 54(3), pp. 384-400, 1991.

[Hei 92] H. J. A. M. Heijmans, Discretization of Morphological Operators, Journal of Visual Communication and Image Representation 3(2), pp. 182-193, 1992. [HY 88] J.G. Hocking, G.S. Young, Topology, Dover Publications Inc., New York, 1988.

[LCG 98] L.J. Latecki, C. Conrad, and A. Gross. Preserving topology by a digitalization process. Journal of Mathematical Imaging and Vision, Vol. 8, pp. 131-159, (1998).

[RT 98] C. Ronse, M. Tajine, Discretization in Hausdorff Space, In preparation, 1998.

[Sch 98] M. Schmitt, Digitalization and connectivity. ISMM'98, Kluwer Academic Publishers, pp. 91-98, 1998.

[Sto 91] J. Stoer, C. Witzgall, Convexity and Optimization in Finite Dimension I., Springer-Verlag, pp. 131-133, 1970.

[Wag 97] D. Wagner, Distance de Hausdorff et Problème Discret-Continu, Mémoire de D.E.A. d'Informatique, U.L.P., Strasbourg, Juin 1997.

[WTR 98] D. Wagner, M. Tajine, C. Ronse, An approach to discretization based on the Hausdorff metric, ISMM'98, Kluwer Academic Publishers, pp. 67-74, 1998. 